\title{
Intrauterine SARS-CoV-2 infection - a case confirming transplacental transmission followed by divergence of the viral genome
}

Mehreen Zaigham ( $\square$ mehreen.zaigham@med.lu.se)

Lund University https://orcid.org/0000-0003-0129-1578

Anna Holmberg

Lund University

Maria Lind Karlberg

Public Health Agency of Sweden

Oskar Karlsson Lindsjö

Public Health Agency of Sweden

Ligita Jokubkiene

Lund University

Johan Sandblom

Lund University

Anna Söderlund Strand

Lund University

Ola Andersson

Lund University

Stefan R. Hansson

Lund University

David Gisselsson Nord

Lund University

Philip Tannenberg

Lund University

\section{Case Report}

Keywords: Cesarean section, COVID-19, intrauterine transmission, hypoxia, placental insufficiency, SARS-CoV-2, transplacental transmission, vertical transmission

Posted Date: October 23rd, 2020

DOl: https://doi.org/10.21203/rs.3.rs-96244/v1

License: (c) (i) This work is licensed under a Creative Commons Attribution 4.0 International License. Read Full License 


\section{Abstract}

Pregnant women and their newborns may be at particular risk for poor outcomes due to the coronavirus (COVID-19) pandemic. Intrauterine SARS-CoV-2 transmission has been suggested but most reports have not ruled out postpartum infection. We demonstrate a confirmed case of transplacental transmission of SARS-CoV-2 in a neonate born to a mother infected in the third trimester. Comprehensive virological, pathological and genetic investigations establish that intrauterine SARS-CoV-2 transmission occurred via the following chain of events; I) maternal viremia in a seemingly mildly symptomatic patient, II) high viral load in the placenta with massive perivillous fibrin deposition, acute intervillositis in areas with strong positivity for SARS-CoV-2 and chorangiosis in the areas less affected by infection and inflammation, III) intrauterine fetal distress with pathological cardiotocography and acidemia in validated umbilical cord blood gases and IV) mild neonatal COVID-19. Whole genome sequencing of isolates from the mother and placenta revealed a single variant of the virus. Interestingly, the neonate displayed a mixed SARSCoV-2 population, harboring both an identical strain to the mother as well as a population with one single-nucleotide polymorphism difference, indicating intrapatient genetic drift.

\section{Highlights}

\section{CONDENSATION}

Transplacental transmission of SARS-CoV-2 with rapid placental dysfunction and fetal distress, followed by confirmed neonatal COVID-19 and divergence of the viral genome.

\section{AJOG AT A GLANCE}

1. Pregnant women and their fetuses may be particularly susceptible to negative outcomes during the COVID-19 pandemic. Intrauterine SARS-CoV-2 transmission has been suggested but rarely proven.

2. Maternal viremia and placental infection led to rapid placental insufficiency followed by intrauterine fetal hypoxia. Whole genome sequencing of isolates from the mother and placenta revealed a single variant of the virus. However, the neonate displayed a mixed SARS-CoV-2 population, harboring both an identical strain to the mother as well as a population with one single-nucleotide polymorphism difference, indicating intrapatient genetic drift in the unique setting of intrauterine transmission.

3. COVID-19 during pregnancy may cause severe placental dysfunction and fetal compromise which needs to be taken in to consideration when outlining guidelines for the surveillance and management of pregnant women with SARS-CoV-2.

\section{Introduction}

The pandemic caused by the emergence of the severe acute respiratory syndrome coronavirus-2 (SARS-CoV-2), causing a novel infection (COVID-19) in humans, has come at a tremendous cost to society and the healthcare sector in particular ${ }^{1}$. Pregnant women are more likely to need intensive care treatment related to COVID-19 as compared to non-pregnant women of reproductive age ${ }^{2}$. COVID-19 infection has also been associated with a higher rate of preterm birth, preeclampsia, Cesarean section (CS), and perinatal death $^{3}$. Neonatal COVID-19 infection are rarely reported and the possibility of a maternal-fetal transmission has previously not been clearly shown. A number of reports have suggested postpartum transmission ${ }^{4}$, but to the best of our knowledge, only Vivanti et al. ${ }^{5}$ have convincingly reported a case of transplacental SARS-CoV-2 transmission. SARS-CoV-2 may be physically blocked by the placental barrier defense mechanisms, combated by immune-regulatory molecular pathways or, in the case of placental infection, mitigate a fierce immune response that may potentially reduce the risk for fetal SARS-CoV- 2 transmission ${ }^{6}$. The placenta is therefore of key interest in understanding perinatal transmission. 
We present a comprehensive case study demonstrating the transplacental transmission of SARS-CoV-2 with rapid placental dysfunction and fetal distress, followed by confirmed neonatal COVID-19.

\section{Results}

\section{Case presentation}

A 27-year-old woman (gravida 2, para 1) was transported with ambulance to the regional university hospital in gestational week (GW) $34+4$ due to a three-day history of fever, abdominal pain and reduced fetal movements. She had developed a dry cough one day prior to the admission.

The woman, an immigrant of Turkish descent did not speak Swedish, was slightly overweight (BMI $\left.27 \mathrm{~kg} / \mathrm{m}^{2}\right)$ but otherwise healthy. She had normal antenatal controls during the current pregnancy. In 2016, she had a normal vaginal delivery in Turkey which was complicated by postpartum hemorrhage. During the current pregnancy, an obstetric ultrasound at GW $32+2$ showed a normal fetal weight deviation of $+8 \%$.

At admission, the patient was promptly isolated in a negative pressure room at the delivery unit and standard operating procedures and personal protective equipment (PPE) were used ${ }^{8}$. A combined nasopharynx (NPH) throat swab for SARS-CoV-2 real time reverse transcriptase quantitative polymerase chain reaction (RT qPCR) was obtained and normal vital parameters (apart from fever 38.3 degrees Celsius) were registered.

The admission cardiotocograph (CTG) test showed reduced baseline variability, absence of accelerations with recurrent prolonged, and late decelerations (Figure 1). In light of the pathological CTG pattern, the obstetric team made the prompt decision to terminate the pregnancy by an immediate-emergency CS. Within eight minutes, the patient was anesthetized and intubated. An uncomplicated CS was performed in an operating theatre with negative pressure in line with the international recommendations for COVID- $19^{8}$. The total blood loss was $200 \mathrm{~mL}$. The amniotic fluid was of normal amount and there were no signs of meconium staining, premature rupture of the amniotic membranes or blood in the amniotic fluid. The placenta was easily detached.

The neonate was immediately transported to a separate room by a designated midwife equipped with sterile clothing and gloves. The neonate showed no initial signs of spontaneous breathing and was ventilated by neonatal staff. A maximum of $80 \%$ supplemental oxygen was needed to maintain adequate saturation. At six minutes of age, the neonate established spontaneous breathing and continuous positive airway pressure $\left(5 \mathrm{~cm} \mathrm{H}_{2} \mathrm{O}\right)$ was maintained for an additional 24 minutes, whereafter further ventilatory support was not needed. At one minute of age, the neonate had an Apgar score of 1 (heart rate $=1$, remaining items $=0$ ), at five minutes of age Apgar 4 (heart rate $=2$, muscular tonus $=1$, reflex irritability $=1$, remaining items $=0$ ), and at ten minutes of age Apgar 8 (heart rate $=2$, respiratory activity $=2$, skin color $=1$, muscular tonus $=2$, reflex irritability $=1$ ). Validated umbilical cord blood gases ${ }^{9}$ from both cord artery and vein were retrieved showing a cord arterial pH of 7.20 and lactate $11 \mathrm{mmol} / \mathrm{L}$

(Supplementary table 2). The birth weight was appropriate for gestational age. Figure 2 illustrates the timeline of events for mother and child.

\section{Maternal clinical course}

After the CS, the mother was isolated in the postpartum ward and the NPH/throat swab taken upon admission returned positive for SARS-CoV-2. Analysis of maternal blood was also RT qPCR positive for SARS-CoV-2 (Table 1). Serology from the day of delivery revealed that the mother was weakly positive for immunoglobulin (Ig) $\mathrm{M}$ and negative for $\mathrm{Ig} \mathrm{G}$ (Table 2). Other maternal laboratory 
tests are shown in Supplementary table 1 . Along with lymphocytopenia $\left(0.7 \times 10^{9} / \mathrm{L}\right)$ and thrombocytopenia $\left(98 \times 10^{9} / \mathrm{L}\right)$; inflammatory markers including c-reactive protein $(36 \mathrm{mg} / \mathrm{L})$, ferritin $(340 \mu \mathrm{mol} / \mathrm{L})$ and lactate dehydrogenase $(9.5 \mu \mathrm{kat} / \mathrm{L})$ were found to be elevated. The clinical condition of the mother improved and she was discharged four days after delivery with thromboprophylaxis (Tinzaparin 4500 IE subcutaneously once daily) for a total six weeks postpartum. A NPH/throat swab taken at day 9 postpartum returned negative for SARS-CoV-2 (Table 1). By day 11 postpartum, the mother was seropositive for anti-SARSCoV-2 IgM and IgG (Table 2). Breast milk analyzed day 14 postpartum was RT qPCR negative for SARS-CoV-2 (Table 1), and further, at day 35 postpartum, negative for anti-SARS-CoV-2 total immunoglobulin (Table 2).

\section{Infection control measures}

According to current national Swedish guidelines for newborns admitted to neonatal ward and with COVID-19 within the family ${ }^{10}$, a neonate can be cared for together with the parent if the required care and isolation measures are possible to provide. Due to a lack of isolation rooms, the neonate in the current case had no contact with any family member, including the mother, during the first 60 hours of life. Since neither skin-to-skin care nor any other contact with the mother occurred, the neonate was regarded as noninfected, and neonatal staff did not use PPE. In accordance with national guidelines ${ }^{10}$, the neonate was tested for COVID-19 using a NPH swab 48 hours after delivery. This test returned positive for SARS-CoV-2 (Table 1) and the neonate was then regarded as contagious. Infection control routines were initiated to investigate a potential COVID-19 breakout at the neonatal ward but also to rule out the possibility of postpartum transmission. All staff that had tended to the neonate $(n=27)$ and all nearby patients $(n=4)$ were tested for COVID-19. The NPH/throat swabs for SARS-CoV-2 RT qPCR returned negative in all cases (data not shown). Symptom surveillance in this group was continued for a further 14 days but no COVID-19 positive cases were discovered during this time.

\section{Neonatal clinical course}

Isolation was carried out as presented above. The neonate was admitted to the neonatal ward at 30 minutes of age due to transient asphyxia and prematurity. Apart from the initial resuscitation, no ventilatory support or supplementary oxygen was needed. During the first day of life (DOL) blood gas analyses were performed mainly to monitor blood glucose levels (Supplementary table 3). Mild hypoglycemia $(2.4 \mathrm{mmol} / \mathrm{L})$ was found at 6 hours of life and it was managed successfully with oral glucose gel and intensified feeding of formula milk via a nasogastric tube.

As the routine RT qPCR test obtained at 48 hours of age returned positive for SARS-CoV-2 and since the clinical condition of the neonate was stable, the neonate was transferred and united with the mother at the postpartum ward isolation room at DOL 3 (60 hours after birth). Breastfeeding was thereafter initiated and the neonate did not receive any breastmilk before this time point. When the mother was later discharged from the postpartum ward at DOL 4, the neonate was transferred to an isolation room at the pediatric infectious disease ward. The mother was still present and breastfeeding was continued. During the entire hospitalization period, the neonate displayed no upper respiratory tract symptoms nor any signs of neonatal COVID-19. At DOL 5, it was observed that the neonate was undernourished and clinical examination revealed moderate signs of hypovolemia, slight ventilatory distress and a mild systolic heart murmur. Pulmonary auscultatory findings were normal. A chest X-ray revealed no abnormalities (Supplementary figure 1). Blood tests including coagulation, liver and hematological analyses were found to be normal for the gestational age. C-reactive protein was not elevated (Supplementary table 3). Following intensified breastfeeding, the hypovolemia, heart murmur and respiratory distress subsided by DOL 7. The neonate was discharged at DOL 15 and was being fully breastfed. A follow-up control at DOL 35 confirmed that the neonate had no respiratory or feeding problems. The physical examination was normal apart from a small, left-sided, asymptomatic, inguinal hernia and additional blood tests revealed no abnormalities (Supplementary table 3). 
Regarding the neonatal COVID-19 infection, repeated RT qPCR analyses showed the lowest CT-value at DOL 5 where after a gradual increase was seen. By DOL 20, SARS-CoV-2 was not detectable in NPH or throat swabs (Table 1). Further, serology revealed that the neonate was anti-SARS-CoV-2 IgG negative at DOL 7 (IgM not analysed due to lack of material). At DOL 14, IgM was positive and IgG still negative and at DOL 20, the neonate was both IgM and IgG seropositive (Table 2).

\section{Viral genome sequencing}

To determine the genetic clade and to fully investigate the viral genetic similarities, virus isolates from the mother (NPH/throat swab obtained on the day of delivery), and neonate (NPH swab obtained at 48 hours of age, labelled DOL 2, and further at DOL 5) as well as from placental tissue, were sent to the Public Health Agency of Sweden for whole-genome sequencing. Next-generation sequencing of samples produced several full length 29 903bp, SARS-CoV-2 genomes, all belonging to the genetic clade 20B/GR/B.1.1 ${ }^{11}$. All four sequences showed high identity, which is illustrated in a phylogenetic tree (Supplementary figure 2). Further sequencing data analysis identified 12 variant positions in the sequences from isolates of the mother and placenta compared to the SARS-CoV-2 reference genome (NC_045512). These variants were also present in the sequences of the neonate isolates (Table 3). Notably, an additional variant, A107G, was identified in the neonate samples but only present in 67 and $80 \%$, respectively, of the sequences.

\section{Placental pathology}

The placenta was easily detached from the uterus during the CS. The remaining umbilical cord stump had a central insertion, was 9 $\mathrm{cm}$ long with a diameter of $1 \times 1.5 \mathrm{~cm}$ and contained three vessels. The membranes had normal color without signs of meconium staining. The trimmed weight of the placental disc was 342 grams, within the $10^{\text {th }}$ to $90^{\text {th }}$ percentile for GW $34+0$ to $34+6^{12}$. At gross sectioning, fibrin depositions were evident as glistening white-grey-pink confluent lesions, encompassing approximately $50 \%$ of the total placental volume (Figure $3 \mathrm{~A}$ ).

Microscopic examination confirmed the presence of confluent intervillous fibrin depositions accompanied by denudation of the villi from trophoblasts and syncytiotrophoblasts with dislocated syncytiotrophoblasts visible in the fibrin (Figure $3 \mathrm{~B}-\mathrm{C}$ ). There were multiple regions of dense intervillous infiltrates of neutrophilic granulocytes and macrophages (Figure $3 \mathrm{D}$ ). The areas devoid of intervillous fibrin depositions frequently showed chorangiosis (Figure $3 \mathrm{E}$ ). Immunohistochemistry confirmed that the inflammatory cell component of the intervillisotis was dominated by myeloperoxidase positive granulocytes and CD68 positive macrophages with sparse amounts of CD3 and CD20 positive lymphocytes (Figure $3 \mathrm{~F}-\mathrm{G}$ ).

Immunohistochemical detection of SARS-CoV-2 nucleoprotein was strongly positive in the cytoplasm and nucleus of villous cytotrophoblasts and syncytiotrophoblasts in areas with intervillositis and fibrin depositions, with some positive staining in the villous stromal cells (Figure $3 \mathrm{H}-J$ ). In contrast, SARS-CoV-2 nucleoprotein staining was focal or absent in most but not all areas devoid of intervillositis (Figure $3 \mathrm{~K}-\mathrm{L}$ ). Additionally, presence of ribonucleic acid (RNA) virus was confirmed in both cytotrophoblasts and syncytiotrophoblasts by in-situ staining for double stranded RNA (Figure $3 \mathrm{M}$ ). There were no signs of villitis or inflammation in the membranes or umbilical cord. Immunohistochemistry for SARS-CoV-2 nucleoprotein was absent or showed faint signal in the amniotic membranes and the fetal chorionic vessels.

In summary, three main histopathological features were found; I) massive perivillous fibrin deposition, II) acute intervillositis in areas with strong positivity for SARS-CoV-2 and III) chorangiosis in the areas less affected by infection and inflammation. 


\section{Discussion}

Vertical transmission is one of the major complications of viral diseases during pregnancy ${ }^{13}$. The previous coronavirus outbreaks of the SARS-CoV-1 and the Middle East respiratory syndrome coronavirus (MERS-CoV) have indicated that pregnant women and their fetuses are particularly susceptible to poor outcomes ${ }^{14,15}$. The current study presents clinical, genetic, and histopathological evidence of intrauterine SARS-CoV-2 transmission.

Several studies have found SARS-CoV-2 in placental tissue, amniotic fluid and in cord blood ${ }^{16-18}$. To the best of our knowledge, intrauterine transmission has been conclusively proven in one case report by Vivanti et al. ${ }^{5}$, in which the placenta was shown to carry a high viral load and clinical data suggested placental dysfunction. Following delivery, the neonate was critically ill and SARS-CoV-2 infection could be identified by early invasive sampling ${ }^{5}$. In another study, Facchetti et al. ${ }^{19}$ analyzed the placentas from 101 women and found SARS-CoV-2 proteins expressed in only of them. This placenta originated from a COVID-19 pregnant woman whose newborn tested positive for viral RNA soon after birth, however, it was unclear if postpartum neonatal infection had been ruled out. Similarly, severe placental pathology due to COVID-19 has been implicated in another case report that led to adverse neonatal outcome despite mild maternal disease ${ }^{20}$. Transplacental transmission of the virus can therefore have dire intrauterine consequences for the fetus in seemingly mildly symptomatic mothers secondary to masked placental dysfunction.

In the present case, apart from being overweight, the mother did not speak Swedish. Socio-economic risk factors have been associated with more severe COVID-19 due to a general higher prevalence of medical problems ${ }^{21,22}$. The mother presented with classic COVID-19 symptoms including fever and a dry cough ${ }^{23}$ but abdominal pain and reduced fetal movements were also reported. Similar to previous reports, we observed that the clinical condition of the mother improved rapidly after delivery 24,25 . Blood tests taken from the mother on the day of delivery revealed discreet liver injury. Transient elevation of serum aminotransferases has often been observed in severe COVID-19 cases $^{26,27}$, however the exact mechanism for direct SARS-CoV-2 related hepatocyte injury is still unknown. The mother also presented with elevated concentrations of several acute phase proteins including ferritin, procalcitonin and c-reactive protein, indicating systemic inflammation ${ }^{28}$. In addition, at the time of delivery, SARS-CoV-2 RNA was found in the maternal blood and RT qPCR indicated the highest viral load within the placenta. RT qPCR does not produce an exact quantification of viral load as different materials are analyzed. However, the CT-values were clearly the lowest in the placental specimen and histopathological placental analyses indicated high levels of SARS-CoV-2. Viral protein was found in the villous cytotrophoblasts, in the syncytiotrophoblasts and massive perivillous fibrin deposits covered over $50 \%$ of the placenta. Viremia in the blood is rare. According to Wang et al. ${ }^{29}$ SARS-CoV-2 RNA was found in only $1 \%$ of blood samples taken from COVID-19 patients. The placental histopathological changes seen in this case are similar to several previous reports on SARS-CoV-2, as well as SARS-CoV- 1 and MERS-CoV5, 15, 19, 20, 30 .

The neonate in the current case suffered from transient asphyxia attributed to intrauterine hypoxia secondary to placental dysfunction. This was signaled by the pathological CTG registering and validated umbilical cord blood gases revealed a cord arterial and venous $\mathrm{pH}$ well below normal median reference values ${ }^{9}$, along with high cord lactate values ${ }^{31}$. Following initial resuscitation, only standard supportive care of prematurity was needed. No evident signs of COVID-19 were observed. The mild respiratory distress and systolic heart murmur observed at DOL 6 was attributed to hypovolemia and promptly relieved after intensified feeding. Blood tests found no abnormalities and repeated RT QPCR testing revealed the lowest CT-values at DOL 5, suggestive of the highest in the upper respiratory tract at this time point. The CT-values later increased and by DOL 20, SARS-CoV-2 RNA was not detectable. Consistent with the observed viral clearance, neonatal IgM and IgG seroconversion was found. Previous knowledge of immunoglobulin transfer during pregnancy along with new data from the current COVID-19 pandemic confirm that anti-SARS-CoV-2 IgG can pass through the placental barrier whilst IgM does not ${ }^{32,33}$. In the current case, maternal serum was weakly positive for IgM and negative for IgG at the day of delivery. Thus, transplacental transfer of anti-SARS-CoV-2 immunoglobulin was not likely and we therefore conclude that the neonate seroconverted by its own means. The possibility of the neonate acquiring COVID-19 postpartum 
was ruled out by vigorous testing of all staff that had been in contact with the neonate during the first 48 hours of life, as well as surrounding patients and their attendees. Secondary symptom surveillance for two weeks revealed no new cases.

To fully determine viral genome similarities between the mother, neonate and the placenta, whole-genome sequencing was performed. All four isolates revealed 29 903bp SARS-CoV-2 genomes, belonging to the genetic clade 20B/GR/B.1.1. Further analysis of the sequencing data showed that the isolate from mother and placenta had 11 single-nucleotide polymorphisms (SNPS) and one multiple-nucleotide polymorphism (MNP) differences compared to the reference Wuhan genome of SARS-CoV-2. Interestingly, the two neonate isolates, from DOL 2 and DOL 5, both had a mixed population of the virus. In addition to a population of the virus with the same genotype as the isolates from the mother and placenta, the neonate isolates contained another population of virus (80\% in DOL 2 and 67\% in DOL 5) with an additional SNP, e.g. A107G. Intrapatient genetic variation has previously been described in both MERS-CoV and SARS-CoV-2 ${ }^{34,35}$. To the best of our knowledge, this is the first case of ongoing genetic change in neonatal COVID-19 in the unique setting of intrauterine transmission. Possibly, the observed genetic drift is a response to a change in the external environment for the virus. Overall however, all virus isolates from mother, child and the placenta, displayed a clear similarity and shared a majority of the SNP's. The SNPs at position 107, 9871, 23851 and 24292, respectively, are rare in the context of sequenced isolates, and have only been described occasionally. Specifically, the SNP at position 9871 is rare among sequenced isolates in international databases, and has only been described in one additional isolate among data currently available ${ }^{11,36}$ The phylogenetic representation of the viral sequences from mother and neonate show a separation, although not at more than a distance of 0.0003 (distance measured as substitutions per site). These findings are in accordance with the findings that the neonate had a mixed population, with a major population having acquired a single SNP compared to the mother. The closest related sequence (three SNP difference) is from a case collected in Lund, Sweden, during the summer, in July, placing similar but not identical sequences in the geographical vicinity of the patient ${ }^{11}$.

Given these genetic findings and the series of events presented above, along with the marked placental pathology and the high viral load, it can therefore be concluded that the neonate was infected in utero. The two main clinical lessons that can be learnt from the current case are; I) COVID-19 during pregnancy may cause severe placental dysfunction and fetal compromise and II) intrauterine SARS-CoV-2 transmission may not necessarily lead to severe neonatal outcome.

\section{Methods}

\section{Patient sampling}

Blood samples, NPH and throat swabs from the mother and neonate were collected according to clinical praxis. Placental tissue for RT qPCR analysis was obtained by a triangulate section reaching the core of the placental disc and the remaining placenta was kept in formalin. Breastmilk was collected day 14 and 35 postpartum for RT qPCR and Ig analysis. NPH swabs were collected from 27 healthcare workers at the Neonatal ward Skåne University Hospital, Malmö, Sweden. Sample collection, processing and laboratory testing followed guidance from Skåne University Hospital, Department of Clinical Microbiology ${ }^{37}$ and The Swedish Public Health Authority ${ }^{38}$.

\section{$P C R$}

SARS-CoV-2 RNA was detected by RT qPCR or using the Panther Fusion open access platform (Hologic, Belgium), in both cases with primers and probe targeting the envelope gene designed by Corman et al. ${ }^{39}$.The RT qPCR was performed with the following modifications: the thermal cycling program was $48^{\circ} \mathrm{C}$ for 10 minutes and $95^{\circ} \mathrm{C}$ for 10 minutes followed by 45 cycles of $95^{\circ} \mathrm{C}$ for 15 seconds and $55^{\circ} \mathrm{C}$ for 45 seconds. All RT qPCR were performed using the Path-ID multiplex one-step RT PCR kit and the Applied Biosystems 7500 fast instrument (Thermo Fisher, USA). 
Virus isolates from NPH/throat swab from the mother, NPH swabs from the neonate (obtained at DOL 2 and DOL 5), and placenta, were sent to the Public Health Agency of Sweden. Samples were extracted (MagLEAD system, Precision System Science, Japan) and subjected to RT qPCR analysis of the RdRp gene of SARS-CoV-2 genome ${ }^{39}$. cDNA was synthesized by SuperScript IV VILO Master Mix (Thermo Fisher Scientific) and used as template for target amplification reactions using two primer pools included in the lon AmpliSeq SARS-CoV-2 Research Panel (Thermo Fisher Scientific) together with 12 spike-in primers for the panel. The targeted amplification and subsequent library preparation steps were performed automatically on an lon Chef system (Thermo Fisher Scientific) using lon AmpliSeq Kit for Chef DL8 (Thermo Fisher Scientific). The amplicon libraries were quantified as previously published ${ }^{40}$ and used for template preparation and lon 540 chip loading on an lon Chef system and sequenced on lon GeneStudio S5 Prime system (Thermo Fisher Scientific). Extracted samples were also sequenced and analyzed on the lon Genexus system (Thermo Fisher Scientific) using the assay Ion Ampliseq SARS-CoV-2 Research Assay 2.0.

\section{Sequence analysis}

The resulting data from the sequencing was processed using the torrent mapping alignment program (TMAP), mapping the sequences towards the reference genome of SARS-CoV-2 (Accession no NC_045512) ${ }^{41}$. After initial mapping, a variant call was performed using the Torrent Variant Caller. The consensus sequence for the viruses was acquired by filtering major frequency alleles from the variant call file (VCF) and apply them to the reference sequence, using bcftool ${ }^{42}$. The COVID19AnnotateSnpEff plugin (Thermo Fisher Scientific), was used to generate an annotated list of variants of the samples. The two sequences were compared using both the VCF results as well as phylogenetically, both by classification by Nextstrain, GSAID and Pangolin classification systems as well as by constructing a phylogenetic tree, using a whole-genome alignment by multiple alignment using fast fourier transform (MAFFT) ${ }^{43}$ and tree construction by IQ-tree ${ }^{44}$.

\section{Phylogenetic tree}

Sequences (hCoV-19/Singapore/705/2020 (EPI_ISL_498603), hCoV-19/Sweden/20-08935/ 2020 (EPI_ISL_534227), hCoV19/Switzerland/AG-ETHZ-270046/2020 (EPI_ISL_541402), hCoV-19/Sweden/20-52588/2020 (EPI_ISL_534256), hCoV19/Sweden/20-52079/2020 (EPI_ISL_510876), hCoV-19/Sweden/20-51044/2020, hCoV-19/Switzerland/ZH-ETHZ-270143/ 2020 (EPI_ISL_541525), hCoV-19/Netherlands/NoordBrabant_157/2020 (EPI_ISL_461192) with close SNP similarity and the reference sequence hCoV-19/Wuhan/WH04/2020 (EPI_ISL_406801) were selected from the global database GISAID and aligned with the four sequences from the mother, neonate and placenta, using MAFFT. Using IQ-TREE 2, a substitution model was established, TPM2+F and a tree was constructed. Branch support was assessed by ultrafast bootstrap approximation, 1000 replicates.

\section{Serology}

In serum, the assessment of COVID-19 antibodies was performed using both the VITROS Immunodiagnostic Products Anti-SARSCoV-2 IgG test Ortho-Clinical Diagnostics Vitros ${ }^{44}$ and ZetaGene COVID-19 Antibody Test IgM/lgG (ZetaGene, Sweden; www.zetagene.com $)^{45}$. For breast milk, the assessment of COVID-19 antibodies was performed using Elecsys, Cobas, Roche AntiSARS-CoV-2 (https://diagnostics.roche.com/) ${ }^{44}$.

\section{Histopathology and immunohistochemistry}

The placenta was subjected to histopathological examination, including careful dissection and macroscopic, and microscopic of the cord, membranes and placental discs. Hematoxylin-eosin staining was done according to routine automated procedures. Immunohistochemistry was by standard methods for myeloperoxidase using a polyclonal antibody (Catalog no. A0398, Dako) at 
1:2500, for CD68 clone PG-m1 (M0786, Dako) at 1:100, for CD3 the clone 2GVG (760-4341, Ventana) prediluted, for CD20 the clone L26 (760-2531, Ventana) prediluted, for SARS-CoV-2 nucleoprotein a polyclonal antibody (40143-T62, Sino Biological) at 1:2000, and for double stranded RNA the clone J2 (10010200, Scicons) at 1:300.

\section{DATA AVAILABILITY}

Sequencing data is available from the European Nucleotide Archive under accession PRJEB40434. All other data generated or analyzed during this study are included in the article.

\section{Declarations}

\section{ACKNOWLEDGMENTS}

The authors would like to thank the COVID-19 in Pregnancy and Early Childhood (COPE) study for allowing the use of a maternal blood sample, Dr Yang De Marinis for donating a COVID-19 serological kit from ZetaGene, Dr Ylva Lindroth and Dr David Nygren from Department of Clinical Microbiology, Skåne University Hospital Lund, Sweden. The authors would like to acknowledge Mia Brytting at the Public Health Agency of Sweden for assistance in the interpretation of sequencing results as well as Niklas Edner for preliminary clinical consulting and facilitation of whole genome sequencing. The authors also gratefully acknowledge the researchers from the originating laboratories responsible for obtaining the specimens and the submitting laboratories where genetic sequence data were generated and shared via the GISAID initiative ${ }^{12}$, on which this research is based.

\section{AUTHOR CONTRIBUTIONS}

M.Z, A.M.H and P.T conceived the project, performed the literature search, prepared the tables, figures, merged and interpreted all the data and wrote the manuscript draft. L.J, M.Z and A.M.H managed the mother. M.Z interpreted the maternal clinical picture and laboratory tests. P.T, J.S, and O.A managed the neonate, interpreted the neonatal clinical picture and laboratory tests. A.S.S interpreted the SARS-CoV-2 diagnostic data. M.L.K and O.K.L performed the whole genome sequencing and data analysis. S.R.H, D.G.N, M.L.K and O.K.L helped in data interpretation and revision of the manuscript. D.G.N performed the pathological examination, prepared the figures and co-authored the text. All authors critically reviewed the manuscript for important intellectual content and approved it in its final version.

\section{COMPETING INTERESTS}

The authors declare no competing interests.

\section{ETHICS}

The mother and father have provided written informed consent to publication, available upon request. The case study was performed in agreement with principles of the Declaration of Helsinki.

\section{MATERIALS \& CORRESPONDENCE}

Correspondence to Mehreen Zaigham: mehreen.zaigham@med.lu.se

\section{FUNDING}


MZ was supported by research grants from Region Skåne and the Medical Faculty, Lund University, Sweden (ALF). The funders had no role in study design, data collection and analysis, decision to publish, or preparation of the manuscript.

\section{References}

1. Ortiz-Prado, E. et al. Clinical, molecular, and epidemiological characterization of the SARS-CoV-2 virus and the Coronavirus Disease 2019 (COVID-19), a comprehensive literature review. Diagn Microbiol Infect Dis 98, 115094 (2020).

2. Allotey, J. et al. Clinical manifestations, risk factors, and maternal and perinatal outcomes of coronavirus disease 2019 in pregnancy: living systematic review and meta-analysis. BMJ 370, m3320 (2020).

3. Di Mascio, D. et al. Outcome of coronavirus spectrum infections (SARS, MERS, COVID-19) during pregnancy: a systematic review and meta-analysis. Am J Obstet Gynecol 2(2), (2020).

4. Molloy, EJ. et al. Neonates in the COVID-19 pandemic. Pediatric research (2020).

5. Vivanti, AJ, et al. Transplacental transmission of SARS-CoV-2 infection. Nat Commun 11, 3572 (2020).

6. Kreis, NN. et al. A Message from the Human Placenta: Structural and Immunomodulatory Defense against SARS-CoV-2. Cells 9 (2020).

7. Marsál, K. et al. Intrauterine growth curves based on ultrasonically estimated foetal weights. Acta Paediatr 85(7), 843-8 (2020).

8. Coronavirus (COVID-19) Infection in Pregnancy. Information for healthcare professionals Version 7: Published Thursday 9 April 2020. Assessed September 25, 2020. Available online: https://www.rcog.org.uk/globalassets/documents/guidelines/2020-0409-coronavirus-covid-19-infection-in-pregnancy.pdf

9. Zaigham, M. et al. Gestational age-related reference values for Apgar score and umbilical cord arterial and venous $\mathrm{pH}$ in preterm and term newborns. Acta Obstet Gynecol Scand 98(12), 1618-1623 (2019).

10. Swedish Society for Obstetrics and Gynecology and Swedish Neonatal Society's recommendations for care of pregnant women and neonates born to women with confirmed or suspected COVID-19. Version 2, updated 2020-04-05. Available at: https://neo.barnlakarforeningen.se/wp-content/uploads/sites/14/2020/03/Rekommendation-om-handläggning-av-gravida-ochbarn-till-kvinnor-med-verifieradelsannolik-Covid-19_ver-2_200405.pdf

11. Global Initiative on Sharing All Influenza Data (GISAID). Genomic epidemiology of hCoV-19 EpiCov. Cited 2020-10-09. Available from: https://www.epicov.org/

12. Kraus, F. et al. Placental Pathology. American Registry of Pathology (2005).

13. Pereira, L. Congenital Viral Infection: Traversing the Uterine-Placental Interface. Annu Rev Viro/ 5, 273-299 (2018).

14. Wong, SF. et al. Pregnancy and perinatal outcomes of women with severe acute respiratory syndrome. Am J Obstet Gynecol 191, 292-297 (2004).

15. Alfaraj, SH. et al. Middle East Respiratory Syndrome Coronavirus (MERS-CoV) infection during pregnancy: Report of two cases \& review of the literature. J Microbiol Immunol Infect 52, 501-503 (2019).

16. Algarroba, GN. et al. Confirmatory evidence of the visualization of severe acute respiratory syndrome coronavirus 2 invading the human placenta using electron microscopy. Am J Obstet Gynecol (2020).

17. Hosier, H. et al. SARS-CoV-2 infection of the placenta. The Journal of clinical investigation 130, 4947-4953 (2020).

18. Patane, L. et al. Vertical transmission of coronavirus disease 2019: severe acute respiratory syndrome coronavirus 2 RNA on the fetal side of the placenta in pregnancies with coronavirus disease 2019-positive mothers and neonates at birth. Am J Obstet Gynecol 2, 100145 (2020).

19. Facchetti, F. et al. SARS-CoV2 vertical transmission with adverse effects on the newborn revealed through integrated immunohistochemical, electron microscopy and molecular analyses of Placenta. EBioMedicine. 59,102951 (2020).

20. Schoenmakers, S. et al. SARS-CoV-2 placental infection and inflammation leading to fetal distress and neonatal multi-organ failure in an asymptomatic woman. medRxiv 2020.06.08.20110437 (2020).

21. Pierce-Williams, J. et al., Clinical course of severe and critical COVID-19 in hospitalized pregnancies: a US cohort study. Am. J. Obstet. Gynecol (2020).

22. Williamson, EJ. et al. Factors associated with COVID-19-related death using OpenSAFELY. Nature 584, 430-436 (2020). 
23. Zaigham, M. et al. Maternal and perinatal outcomes with COVID-19: A systematic review of 108 pregnancies. Acta Obstet Gynecol Scand 99, 823-829 (2020).

24. Ronnje, L. et al. Complicated COVID-19 in pregnancy: a case report with severe liver and coagulation dysfunction promptly improved by delivery. BMC Pregnancy Childbirth 20, 511 (2020).

25. Kolkova, Z. et al. Obstetric and intensive-care strategies in a high-risk pregnancy with critical respiratory failure due to COVID-19: A case report. Case Rep Womens Health 27, e00240 (2020).

26. Huang, C. et al. Clinical features of patients infected with 2019 novel coronavirus in Wuhan, China. Lancet 395:497-506 (2020)

27. Wang, D. et al. Clinical characteristics of 138 hospitalized patients with 2019 novel coronavirus-infected pneumonia in Wuhan, China. JAMA (2020).

28. Henderson, LA. et al. On the alert for cytokine storm: Immunopathology in COVID-19. Arthritis Rheumatol (2020).

29. Wang, W. et al. Detection of SARS-CoV-2 in Different Types of Clinical Specimens. JAMA 323(18):1843-1844 (2020).

30. WF, Ng. et al. The placentas of patients with severe acute respiratory syndrome: a pathophysiological evaluation. Pathology 38(3), 210-218 (2006).

31. Wiberg, N. et al. Lactate concentration in umbilical cord blood is gestational age-dependent: a population-based study of 17867 newborns. BJOG 115, 704-709 (2008).

32. Kohler, PF. et al. Elevation of cord over maternal IgG immunoglobulin: evidence for an active placental IgG transport. Nature 210, 1070-1071 (1966).

33. Zeng, H. et al. Antibodies in Infants Born to Mothers With COVID-19 Pneumonia. JAMA (2020).

34. Park, D. et al. Analysis of intrapatient heterogeneity uncovers the microevolution of Middle East respiratory syndrome coronavirus. Cold Spring Harb Mol Case Stud. 2(6), a001214 (2016).

35. Jary, A. et al. Evolution of viral quasispecies during SARS-CoV-2 infection. Clin Microbiol Infect (2020).

36. GenBank [Internet]. Bethesda (MD): National Library of Medicine (US), National Center for Biotechnology Information; 2004. Cited 2020-10-09. Available from: https://www.ncbi.nlm.nih.gov/genbank/

37. Instructions for sampling and analysis of COVID-19. Department of Laboratory Medicine, University Hospital, Sweden. Updated 2020-10-06. Available at: https://vardgivare.skane.se/vardriktlinjer/laboratoriemedicin/provtagning-och-analys-av-covid19/\#165894

38. Recommendations for laboratory handling of patient samples with COVID-19. The Swedish Public Health Authority. Updated 2020-09-22. Available at: https://www.folkhalsomyndigheten.se/smittskydd-beredskap/utbrott/aktuella-utbrott/covid19/information-till-varden/rekommendationer-for-laboratoriehantering-av-patientprover-med-avseende-pa-covid-19/

39. Corman, VM. et al. Detection of novel coronavirus (2019-nCoV) by real-time RT-PCR. Euro Surveill 25 (2020).

40. Beser, J. et al. Improving the genotyping resolution of Cryptosporidium hominis subtype IbA10G2 using one step PCR-based amplicon sequencing. Infect Genet Evol 55, 297-304 (2017)

41. Wu, F. et al. A new coronavirus associated with human respiratory disease in China. Nature 579, 265-269 (2020)

42. Li, H. et al. The Sequence Alignment/Map format and SAMtools. Bioinformatics 25, 2078-2079 (2009).

43. Katoh, K. et al. MAFFT: a novel method for rapid multiple sequence alignment based on fast Fourier transform. Nucleic Acids $\operatorname{Res}$ 30, 3059-3066 (2002).

44. Nguyen, LT. et al. IQ-TREE: a fast and effective stochastic algorithm for estimating maximum-likelihood phylogenies. Mol Biol Evol 32, 268-274 (2015).

45. Ravi, N. et al. Diagnostics for SARS-CoV-2 detection: A comprehensive review of the FDA-EUA COVID-19 testing landscape. Biosens Bioelectron 165, 112454 (2020).

46. Yang, D. M. et al. Serology assessment of antibody response to SARS-CoV-2 in patients with COVID-19 by rapid IgM/lgG antibody test. Preprint. doi: https://doi.org/10.1101/2020.08.05.20168815

\section{Tables}

Table 1. 
Real time reverse transcriptase quantitative polymerase chain reaction (RT qPCR) analyses for severe acute respiratory syndrome coronavirus-2 (SARS-CoV-2) in maternal samples, placental sample, and neonatal samples.

\section{Maternal samples}

\begin{tabular}{|llll|}
\hline Sample & Time of sampling & Result & CT-value \\
\hline Duo swab (NPH/throat) & Day of delivery & Positive & 26.7 \\
\hline Blood (EDTA-plasma) & Day of delivery & Positive & 31.7 \\
\hline Duo swab (NPH/throat) & Day 9 postpartum & Negative & - \\
\hline Breastmilk & Day 14 postpartum & Negative & - \\
\hline
\end{tabular}

Placental sample

\begin{tabular}{|llll|}
\hline Sample & Time of sampling & Result & CT-value \\
\hline Placenta & Day of delivery & Positive & 13.6 \\
\hline
\end{tabular}

\section{Neonatal samples}

\begin{tabular}{|llll|}
\hline Sample & Time of sampling & Result & CT-value \\
\hline NPH swab & DOL 2 & Positive & 27.9 \\
\hline NPH swab & DOL 5 & Positive & 18.1 \\
\hline Throat swab & DOL 5 & Positive & 23.5 \\
\hline NPH swab & DOL 12 & Positive & 36.1 \\
\hline Throat swab & DOL 12 & Positive & 33.3 \\
\hline NPH swab & DOL 14 & Positive & 34.5 \\
\hline Throat swab & DOL 14 & Negative & - \\
\hline NPH swab & DOL 16 & Positive & 29 \\
\hline Throat swab & DOL 16 & Negative & - \\
\hline NPH swab & DOL 20 & Negative & - \\
\hline Throat swab & DOL 20 & Negative & - \\
\hline
\end{tabular}

\section{Table 2.}

Serological analyses for severe acute respiratory syndrome-2 (SARS-CoV-2) immunoglobin (Ig) M, IgG, and total lg (i.e. $\lg A$, IgM and $\mathrm{lgG}$ ) in maternal samples and neonatal samples.

\section{Maternal samples}




\begin{tabular}{|llll|}
\hline Sample & Time of sampling & Ig class & Result \\
\hline Serum & Day of delivery & $\operatorname{lgM}$ & Weak positive \\
\hline Serum & Day of delivery & $\operatorname{lgG}$ & Negative \\
\hline Serum & Day 12 postpartum & $\operatorname{lgM}$ & Positive \\
\hline Serum & Day 12 postpartum & $\operatorname{lgG}$ & Positive \\
& & & \\
\hline Breastmilk & Day 35 postpartum & Total lg & Negative \\
\hline
\end{tabular}

\section{Neonatal samples}

\begin{tabular}{|llll|}
\hline Sample & Time of sampling & Ig class & Result \\
\hline Serum & DOL 7 & IgG & Negative \\
\hline Serum & DOL 14 & IgM & Positive \\
\hline Serum & DOL 14 & IgG & Negative \\
\hline Serum & DOL 20 & IgM & Positive \\
\hline Serum & DOL 20 & IgG & Positive \\
\hline
\end{tabular}

\section{Table 3.}

Variant analysis and annotation of whole-genome sequencing data from virus isolates obtained from the mother and placenta at delivery and from the neonate at day of life (DOL) 2 and DOL 5. The table shows all identified variants, single-nucleotide polymorphisms (SNP) or multiple-nucleotide polymorphisms (MNP), with corresponding nucleotide position in reference genome of for severe acute respiratory syndrome coronavirus-2 (SARS-CoV-2) (NC_045512), the frequency (\%) of the variant in the sequencing data and their gene-based annotation. 


\begin{tabular}{|c|c|c|c|c|c|c|c|c|c|}
\hline \multirow[t]{2}{*}{ Variant } & \multicolumn{4}{|c|}{$\%$ variant in the sample } & \multirow{2}{*}{$\begin{array}{l}\text { Type } \\
\text { of } \\
\text { variant }\end{array}$} & \multirow[t]{2}{*}{ Gene } & \multirow{2}{*}{$\begin{array}{l}\text { Type of } \\
\text { mutation }\end{array}$} & \multirow[t]{2}{*}{ Affected AA } & \multirow{2}{*}{$\begin{array}{l}\text { Predicted } \\
\text { consequence* }\end{array}$} \\
\hline & Mother & Placenta & $\begin{array}{l}\text { DOL } \\
2\end{array}$ & $\begin{array}{l}\text { DOL } \\
5\end{array}$ & & & & & \\
\hline A107G & 0 & 0 & 80 & 67 & SNP & orf1ab & $\begin{array}{l}\text { upstream } \\
\text { gene }\end{array}$ & . & MODIFIER \\
\hline C241T & 100 & 100 & 100 & 100 & SNP & orf1ab & $\begin{array}{l}\text { upstream } \\
\text { gene }\end{array}$ & . & MODIFIER \\
\hline C3037T & 100 & 100 & 100 & $>99$ & SNP & orf1ab & synonymous & Phe924Phe & LOW \\
\hline G9871A & 100 & 100 & $>99$ & 100 & SNP & orf1ab & synonymous & Thr3202Thr & LOW \\
\hline C14408T & 100 & $>99$ & 100 & 100 & SNP & orf1ab & missense & Pro4715Leu & MODERATE \\
\hline A23403G & 100 & 100 & 100 & 100 & SNP & S & missense & Asp614Gly & MODERATE \\
\hline A23851G & 100 & 100 & 100 & 100 & SNP & $S$ & synonymous & Leu763Leu & LOW \\
\hline A24292G & 100 & $>99$ & 100 & 100 & SNP & $S$ & synonymous & Gly910Gly & LOW \\
\hline C26447T & 100 & 100 & 100 & 100 & SNP & E & missense & Ser68Phe & MODERATE \\
\hline G26727T & 100 & 100 & 100 & 100 & SNP & $M$ & missense & Ala69Ser & MODERATE \\
\hline GGG28881AAC & 100 & 100 & 100 & 100 & MNP & $\mathrm{N}$ & missense & ArgGly203LysArg & MODERATE \\
\hline C28887T & $>99$ & $>96$ & $>99$ & $>99$ & SNP & $\mathrm{N}$ & missense & Thr205Ile & MODERATE \\
\hline C29666T & 100 & 100 & 100 & 100 & SNP & ORF10 & missense & Leu37Phe & MODERATE \\
\hline
\end{tabular}

*Ensemble variation. Calculated variant consequences.

http://www.ensembl.org/info/genome/variation/prediction/predicted_data.html

\section{Figures}



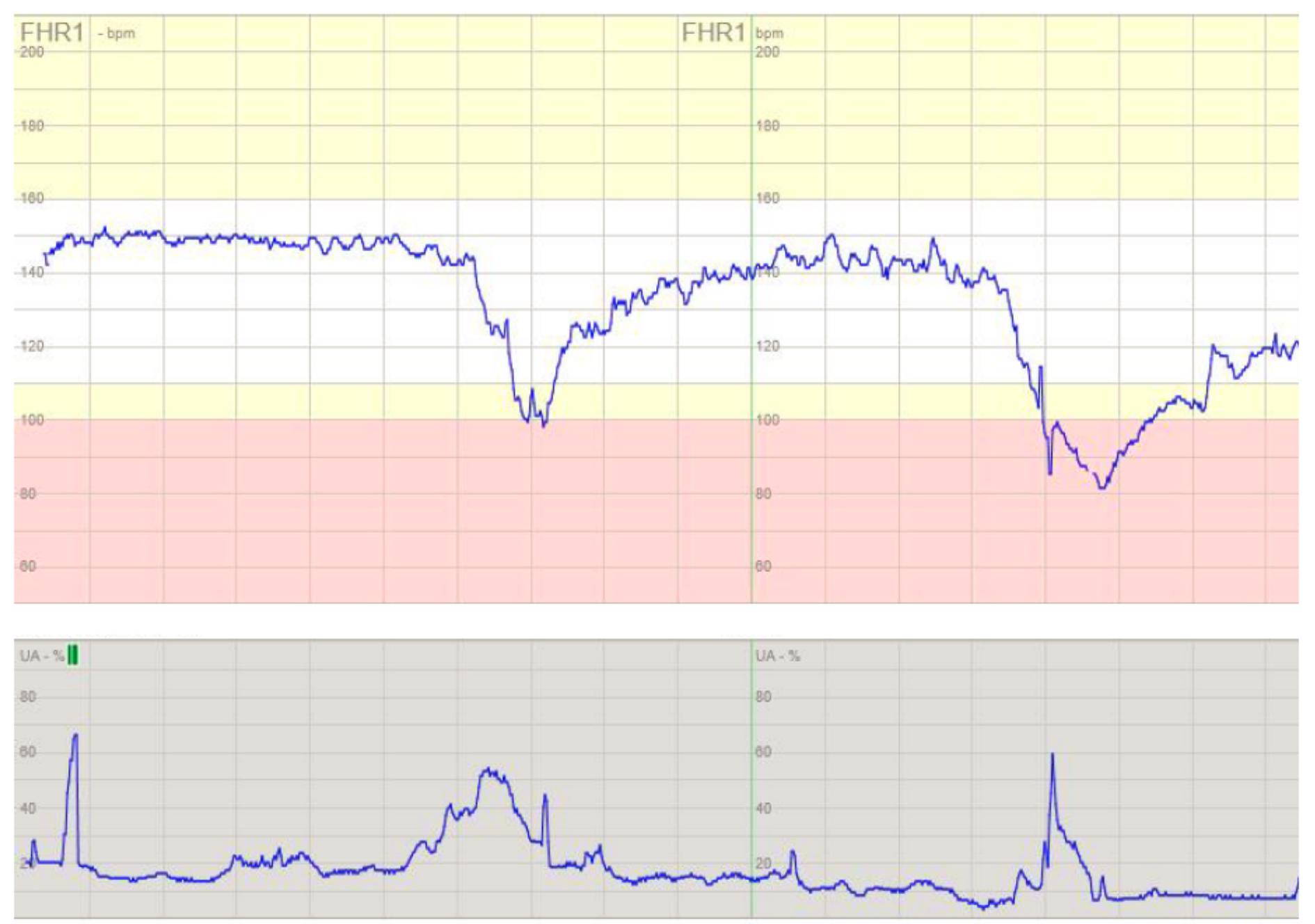

\section{Figure 1}

Admission cardiotocograph (CTG) of the fetal heart rate. Normal fetal heart rate with reduced baseline variability, absence of accelerations and with recurrent prolonged and late decelerations. These findings are suggestive of a pathological category III fetal heart rate tracing which is strongly associated with adverse neonatal outcome. The CTG was recorded 8 minutes before the immediate-emergency cesarean section was performed. 

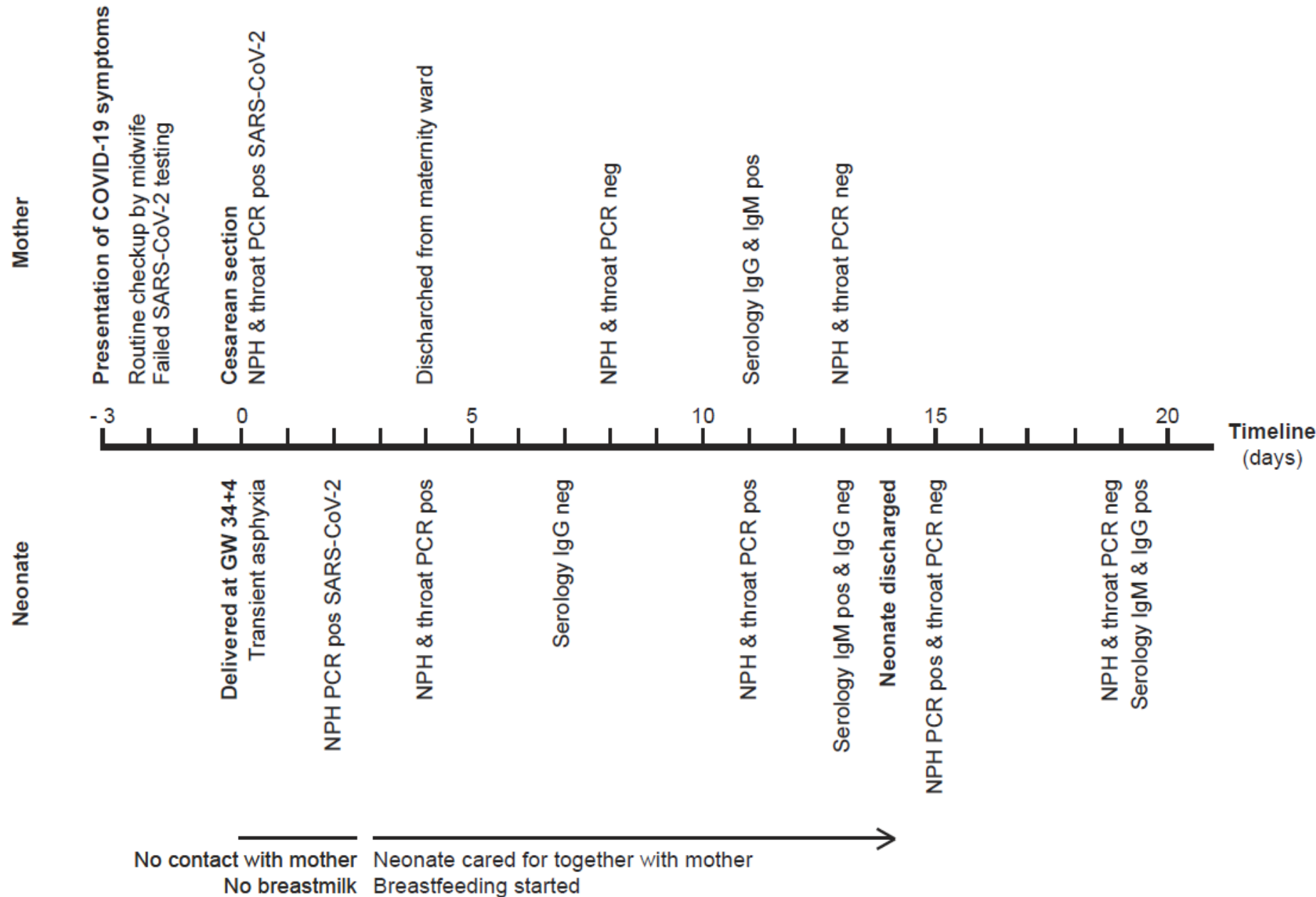

Figure 2

Timeline of events for mother and neonate. SARS-CoV-2; severe acute respiratory syndrome coronavirus-2, PCR; Real time reverse transcriptase quantitative polymerase chain reaction, GW; gestational week, NPH; nasopharynx, Ig; Immunoglobulin. 

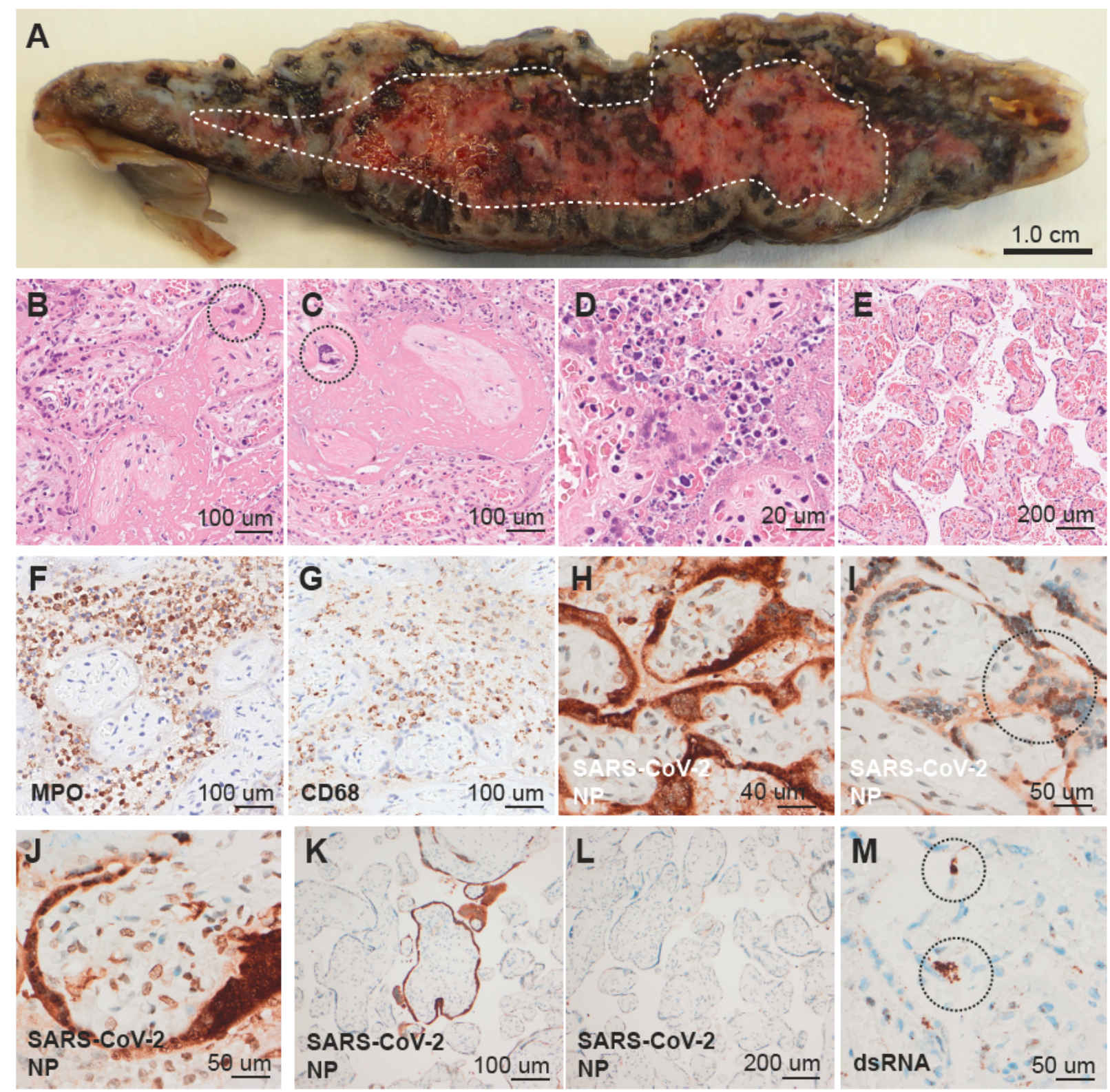

\section{Figure 3}

Placental pathology. (A) Transected placenta with confluent accumulation of fibrin demarcated (white broken line) (B-C) Massive intervillous fibrin deposition surrounding denuded villi with extravillous syncytiotrophoblasts (circles) located in the fibrin (D) Acute intervillositis with karrhyorectic neutrophils in the intervillous space and degeneration of the villous trophoblast layer (E)

Representative region of chorangiosis (F-G) Immunohistochemical staining for myeloperoxidase (MPO) and CD68 with positivity in inflammatory cells in areas of intervillositis (H-J) Severe acute respiratory syndrome coronavirus-2 (SARS-CoV-2) nucleoprotein (NP) detected in nucleus and (circle in I) and cytoplasm in villous trophoblasts and syncytiotrophoblasts as well as in the nucleus of villous stromal cells $(\mathrm{J})$ in areas of intervillositis (K-L) Areas without intervillositis showed absent or focal staining for SARS-CoV-2 nucleoprotein of villi. M. Double stranded RNA (dsRNA) detected in villous trophoblasts and syncytiotrophoblasts (circles).

\section{Supplementary Files}

This is a list of supplementary files associated with this preprint. Click to download.

- SupplTable1Maternallabwork.docx

- SupplTable2Cordbloodgases.docx 
- SupplTable3Mainlaboratoryfindingsintheneonate.docx

- SupplFig1xray.pdf

- SupplFig2Phylogenetictree.pdf 